\title{
Erratum to: Reversible watermarking for 2D vector maps based on normalized vertices
}

\author{
Nana Wang ${ }^{1}$
}

Published online: 28 December 2016

(C) Springer Science+Business Media New York 2016

\section{Erratum to: Multimed Tools Appl \\ DOI: 10.1007/s11042-016-3970-5}

The author regrets that the original version of this article contain four errors. The author did not consider the first vertex and the last vertex of each polygon/polyline during the experiments, the results of proposed method in Table 2, the last column of Tables 7 and 8, and Fig. 5 was wrong. The author, therefore, has corrected errors as follows.

The author would like to apologize for any inconvenience caused.

1. The Maxd and $d$ values of the proposed method in Table 2

Table 2 The Maxd and $d$ values between the original vector maps and the recovered ones

\begin{tabular}{lll}
\hline Vector maps & Maxd $(\mathrm{m})$ & $d(\mathrm{~m})$ \\
\hline M1 & $3.5624 \times 10^{-6}$ & $1.9036 \times 10^{-6}$ \\
M2 & $1.7858 \times 10^{-6}$ & $9.5164 \times 10^{-7}$ \\
M3 & $4.5061 \times 10^{-6}$ & $2.4517 \times 10^{-6}$ \\
M4 & $4.4588 \times 10^{-6}$ & $2.4343 \times 10^{-6}$ \\
Average of 50 vector maps & $3.9563 \times 10^{-6}$ & $1.6598 \times 10^{-6}$ \\
\hline
\end{tabular}

The online version of the original article can be found at http://dx/doi.org/10.1007/s11042-016-3970-5

Nana Wang

wangnana_5@yahoo.com

1 Present address: School of Computer Science and Technology, Jiangsu Normal University, Xuzhou 221116, People's Republic of China 
2. The $d$ values the proposed method in Table 7

Table 7 Invisibility of different methods (m)

\begin{tabular}{lcccccr} 
Vector maps & Cao et al. [22] & Wang et al. [23] & Wang et al. [24] & Peng et al. [38] & Xiao et al. [39] & Proposed \\
\hline M1 & 0.3362 & 0.1342 & 0.1235 & $4.4039 \times 10^{-5}$ & 0.0174 & 0.1611 \\
M2 & 3.0849 & 0.6155 & 0.6266 & 0.0002 & 3.1594 & 0.8055 \\
M3 & 2.9274 & 1.3254 & 1.2464 & 0.0003 & 5.0636 & 1.6145 \\
M4 & 93.7668 & 26.1374 & 24.7573 & 0.0060 & 2.6848 & 31.6390 \\
\hline
\end{tabular}

3. The capacity the proposed method in Table 8

Table 8 Capacity of different methods ( $b p v)$

\begin{tabular}{lllllll}
\hline Vector maps & $\begin{array}{l}\text { Cao et al. } \\
{[22]}\end{array}$ & $\begin{array}{l}\text { Wang et al. } \\
{[23]}\end{array}$ & $\begin{array}{l}\text { Wang et al. } \\
{[24]}\end{array}$ & $\begin{array}{l}\text { Peng et al. } \\
{[38]}\end{array}$ & $\begin{array}{l}\text { Xiao et al. } \\
{[39]}\end{array}$ & Proposed \\
\hline M1 & 0.2395 & 1.9827 & 0.9993 & 0.9993 & 1.9991 & 3.9981 \\
M2 & 0.5737 & 1.8182 & 0.9999 & 0.9999 & 1.9998 & 3.9997 \\
M3 & 0.5347 & 1.9569 & 0.9892 & 0.9999 & 1.9998 & 3.9567 \\
M4 & 0.6061 & 1.9165 & 0.9758 & 0.9933 & 1.9987 & 3.9273 \\
Average of 50 & 0.5210 & 1.8970 & 0.9927 & 0.9988 & 1.9996 & 3.9890 \\
$\quad$ vector maps & & & & & & \\
\hline
\end{tabular}

4. The Fig. 5

Fig. 5 Relationship between average embedding distortion $d$ and $b$

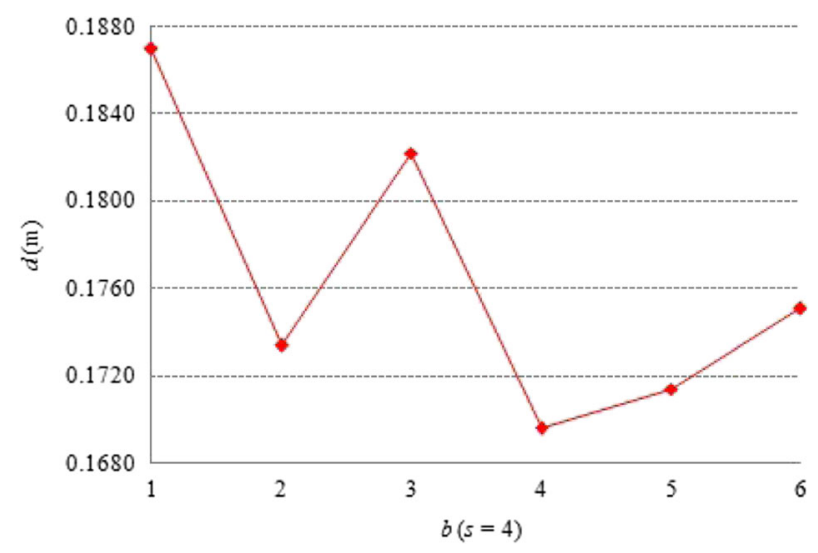

